\title{
El buen profesional ${ }^{1}$
}

\author{
The good professional
}

\author{
Damián Salcedo Megales \\ Universidad Complutense de Madrid (España) \\ dsalcedo@filos.ucm.es
}

Recibido: 05/03/2015

Revisado: $13 / 03 / 2015$

Aceptado: 16/04/2015

Disponible on line: 10/06/2015

\begin{abstract}
Resumen
El artículo parte de una definición formal del juicio moral para determinar el carácter ético de la profesionalidad, entendida como el cumplimiento de un compromiso institucional con ciertos valores fundamentales que guían la actuación profesional. Ese conjunto de valores permite diferenciar al buen profesional del profesional deshonesto o corrupto. Definimos así un concepto de integridad profesional correlacionado con la idea de identidad personal. Ello nos permite argumentar en favor de una nueva manera de entender la relación entre moral personal y moral profesional, la cual implica una nueva forma de concebir la identidad profesional. El caso defendido por Soyer del «derecho a fracasar» de los clientes ilustra lo que esta nueva concepción implicaría.
\end{abstract}

Palabras clave: valores profesionales, integridad, identidad profesional, derecho de fracasar.

\begin{abstract}
This paper starts from a formal definition of moral judgment to determine the ethical character of professionalism, which is understood as the fulfillment of an institutional commitment with some core values which guide the professional performance. Such set of values let us to draw a line between the good professional and the dishonest (or corrupt) professional. We define a notion of professional integrity correlating with personal identity. This allows arguing for a new way of understanding the relationship between personal morality and professional ethics, which implies a new way of thinking about professional identity. These suggestions are illustrated by the discussion of Soyer's case for a client's «right to fail».
\end{abstract}

Keywords: professional values, integrity, professional identity, right to fail.

Referencia normalizada: Salcedo Megales, D. (2015): «El buen profesional». Cuadernos de Trabajo Social, 28(1): $19-26$.

Sumario: 1. El buen profesional. 2. Nota bibliográfica. 3. Referencias bibliográficas.

\section{EI buen profesional}

Nos referimos a un trabajador como un «buen profesional» cuando queremos expresar nuestro aprecio por las cualidades profesionales que ha desplegado al realizar un servicio. La calificación de «buen(o)» en este caso pretende cualificar a todo su ser profesional. Por lo que no decimos que se ha comportado como un «buen profesional». Lo que decimos es que es un «buen profesional». Es probable, no obstante, que nuestro juicio sea precipitado y que, en realidad, lo razonable fuera decir solo que el comportamiento que ha tenido durante el periodo en el que nos hemos relacionado, además del servicio que nos ha prestado, nos han parecido propios de un buen profesional. Lo cierto, sin embargo, es que la confianza que tenemos en nuestra capacidad de juzgar nos lleva a saltarnos todos los pasos intermedios, las cautelas debidas, y a que alcancemos rápidamente lo que ten-

${ }^{1}$ Trabajo realizado en el marco del proyecto FFI2013-46530-P, Toma de decisiones en la práctica profesional del trabajo social, del cual es investigadora principal $\mathrm{M}^{\mathrm{a}} \mathrm{J}$. Úriz Pemán, financiado por el Ministerio de Economía y Competitividad del Gobierno de España. Quiero agradecer a todos los compañeros del grupo de investigación el apoyo que siempre he recibido de ellos. 
dría que ser una conclusión deliberativa, la cual expresamos con la aprobación.

Ahora bien, si alguien nos pidiera que explicásemos los motivos en los que sustentamos tal juicio de aprobación, entonces tendríamos que enumerar todas aquellas cualidades que creemos ha de tener un profesional y mostrar que, efectivamente, el trabajador con el que hemos tenido una relación de servicio las cumplía, concluyendo, entonces, ahora sí, razonadamente, que se debe a tal valoración el que mostremos nuestra aprobación hacia su modo de ser profesional. Si, una vez dadas estas explicaciones, nuestro interlocutor se mostrara en desacuerdo con nosotros, por ejemplo, manifestando que él ha tenido una experiencia distinta con el mismo trabajador y que no le ha parecido muy profesional, entonces esa persona tendría, igualmente, que explicarnos cuáles son las cualidades que ha de tener un profesional y en qué sentido el trabajador no las ha cumplido. Podríamos entrar, de este modo, en un diálogo razonado sobre las cualidades que creemos que ha de tener un profesional y el modo de cumplirlas en una relación de servicio particular. Como resultado de tal diálogo es posible que llegáramos a comprender mejor las cualidades que creemos que se deben utilizar como criterio para determinar la profesionalidad y el modo en que se han de cumplir en la relación profesional, dado que es fácil que nuestro interlocutor haya reparado en aspectos que a nosotros nos hayan pasado inadvertidos y que, después, de la debida reflexión, pensemos que, ciertamente, se han de tener en cuenta. También es posible que, al final, nos pongamos de acuerdo en ellos, aunque sigamos sosteniendo que, en nuestro caso, el trabajador en cuestión los satisfizo, aunque sea posible que en el caso de nuestro interlocutor no lo hiciera. Sin embargo, también es posible que por más que se discuta y se expongan los puntos de vista, los nuestros y los de nuestro interlocutor, tengamos concepciones tan distintas de la profesionalidad que no sea posible que nos pongamos de acuerdo en nada. Es posible. Sobre lo que me interesa llamar la atención es sobre el hecho de que no depende de que nos pongamos de acuerdo, sino más bien de las características que tiene nuestra manera de hacer juicios, de lo que depende el que los podamos llamar morales. Esto es, si el juicio de aprobación que emitimos sobre un profesional ha de inscribirse en el ámbi- to específico de los juicios morales y no en el de los técnicos, o los estéticos o, pongamos por caso, el de los políticos, se debe a ese modo peculiar en que lo hemos razonado.

En primer lugar, creemos que nuestros juicios morales han de tener detrás algún conjunto de razones. Dichas razones serán sólidas en la medida en que creemos que cualquiera que las considere las habría de aceptar y que, por eso mismo, el juicio que se sigue de ellas, tendría que ser aceptable por cualquiera que aceptara las razones que lo avalan. Reflexionamos con nosotros mismos, o dialogamos con los demás, para establecer en qué consistirán unas razones sólidas que valgan en un caso y cuál será el juicio que de modo congruente se seguirá de ellas. Hay muchos modos en los que es posible el desacuerdo. Con todo, a menos que nuestro estado emocional nos la juegue, una vez que los hechos del caso y los criterios que creemos válidos se exponen y se revisan con claridad, nuestro desacuerdo será un desacuerdo moral. Lo que quiero decir es que, aunque nosotros y nuestro interlocutor, al final, no estuviéramos de acuerdo en cómo valorar moralmente la relación de servicio, en lo que sí estaríamos de acuerdo es en comprender que nuestros puntos de vista son puntos de vista morales; que no estamos oponiendo razones estéticas a razones morales, o razones puramente técnicas a razones políticas. El conjunto de ambos razonamientos son reconociblemente morales, por más que los juicios sobre la moralidad del caso sean contrarios.

¿Por qué es así? Desde que empezamos con nuestro apresurado juicio inicial de aprobación hasta que hemos alcanzado nuestro juicio final reflexivo, nuestro modo de enfrentarnos a la discusión ha tenido una característica muy peculiar. En ningún momento hemos intentado sostener que nuestra posición, ni en la discusión ni en la valoración del caso, era especial, mejor, o diferente. Como tampoco, apoyándonos en ese privilegio, hemos intentado ridiculizar la de nuestro interlocutor. Creemos que para que nuestro razonamiento sea un razonamiento valido tendría que ser aceptable por toda persona que lo conociese. Para conseguirlo, hemos intentado que tal razonamiento no se vea influido ni por nuestros gustos particulares ni por nuestros sentimientos especiales ni por cualquier otro rasgo psicológico propio de nuestra individualidad. Por el contrario, hemos deliberada- 
mente intentado que todo eso quedara fuera de nuestro modo de razonar sobre la relación profesional en cuestión. Precisamente, se debe a que hemos intentado proceder de ese modo, el que estemos convencidos de que, explicando bien lo sucedido y los criterios con los que juzgamos, cualquiera estará de acuerdo en juzgar la actuación del profesional del mismo modo. Esta es la segunda característica del razonamiento específicamente moral.

Podemos juzgar las cosas de este mundo desde muchos puntos de vista. Sin embargo, solo lo hacemos moralmente, cuando tratamos de construir un razonamiento que sea aceptable por toda persona que también esté dispuesta a construir un razonamiento sobre el asunto en cuestión. Cuando hemos dicho al principio que el trabajador es un buen profesional podemos motivar nuestro juicio refiriéndonos a su capacidad de ayudarnos a la resolución de un problema, el conocimiento de los recursos necesarios para hacerlo, el sentido del compromiso y el esfuerzo que ha realizado para encontrar una solución, la lealtad mostrada al no habernos abandonado para atender otros asuntos, la valentía para no dejarse intimidar por las dificultades, la honestidad a la hora de exponer la realidad de las dificultades, etc. Creemos que todas ellas son cualidades profesionales valiosas; creemos que el trabajador las ha desplegado en la relación que hemos tenido con él; creemos que el juicio que debemos hacer solo puede ser de aprobación. Todo eso, sin embargo, podría formar parte de una explicación fundada en nuestro modo particular de creer o en cómo nos gustan las cosas o en alguna visión política de la sociedad. Lo que hacemos, entonces, al dar estas explicaciones, es mostrar nuestra satisfacción por cómo son las cosas o por ver realizadas nuestras aspiraciones y lo único que esperamos de nuestro interlocutor es que nos entienda y, quizás, sentirnos acompañados en ellas. Ahora bien, si, por el contrario, estamos convencidos de que, a quien se lo expliquemos, va a entender que de lo que estamos hablando es de moral, entonces nuestra explicación tiene que ser construida como un razonamiento en el que todas aquellas apreciaciones se utilicen de otro modo. Esa característica del razonamiento es lo que lo hace distintivamente moral y es la que se difunde a los juicios que hacemos, a las decisiones que tomamos, o a la conducta que tenemos. El reino de la moral lo que nos pide es que universalicemos nuestro punto de vista.

Los profesionales hablan de ética profesional como de un compromiso personal que tienen de servir a sus clientes según criterios que sean aceptables para todo aquel que quiera enjuiciar moralmente su conducta profesional. A veces se suele explicar la moralidad de la actividad profesional apelando a la vocación sentida por la persona, la cual le ha llevado a formarse y a desempeñar la actividad en cuestión. A veces, también, encontramos que se explica la moralidad de la actividad profesional haciendo referencia a los bienes que procura a la sociedad. Esto es erróneo. Como se deduce de lo explicado, lo que permite calificar de moral la actividad profesional es que se realiza según los principios con los que la profesión se ha comprometido con la sociedad, los cuales serían los criterios que guían su desarrollo y que obligan a toda persona que quiera dedicarse a la profesión. Tal conjunto de principios definen la cualidad moral de la actividad profesional, porque se los ha podido presentar ante la sociedad como un conjunto de principios que cualquier persona dispuesta a examinarlos los podría aceptar racionalmente. En ese sentido, son morales, porque son universalizables. En consecuencia, lo que finalmente hace moral la conducta de un profesional no es la fuerza de su vocación ni los bienes que su actividad procura a sus clientes o a la sociedad en general. Se trata, más bien, de que su actividad realiza el conjunto de principios que su profesión ha proclamado como constitutivos de su profesionalidad y que la sociedad ha aceptado. El significado moral de la conducta profesional se determina, entonces, por medio de un proceso de reconocimiento por parte de la sociedad de lo que la profesión proclama como criterios para juzgar sus actividades. Si son reconocidos, entonces, serán los criterios con los que el profesional decidirá y juzgará tanto la propia conducta como las de los demás profesionales. También serán los que utilizarán los clientes (u otros miembros de la sociedad) para juzgar las actividades profesionales. Serán, asimismo, el asunto sobre el que se debatirá y se discutirá, tanto en las disputas profesionales, como en los foros sociales. Finalmente, serán el objeto sobre el que se establecerán los acuerdos y los desacuerdos, las ortodoxias y las heterodoxias profesionales. To- 
das esas actividades serán característicamente morales en la medida en que sigan alentando el espíritu de universalidad que caracteriza a los principios profesionales.

Muchos profesionales no son del todo conscientes de cuánto depende la calificación ética de su actuación de que ésta se funde y logre expresar de forma concreta el compromiso público que la profesión a la que se pertenece ha hecho con un conjunto de principios, normas y criterios morales. Una persona que se presenta ante sus clientes como profesional crea la expectativa de que se comportará según ciertos criterios. Al decir «soy un profesional» está diciendo me comprometo a comportarme de un cierto modo. Si, finalmente, no lo hace, no estando a la altura de esa expectativa, aparecerá como un hipócrita. Por ejemplo, si uno está comprometido con la honestidad profesional y a la primera dificultad engaña, entonces carece de integridad profesional. En cambio, cuando sistemáticamente se desvirtúan los principios que la profesión se ha comprometido a seguir ante la sociedad como guía de su ejercicio, la conducta se califica según esa especie particular de inmoralidad que es la corrupción. Hipocresía, falta de honestidad y de integridad, corrupción moral, en el caso de los profesionales, remiten siempre a un mundo compartido de principios (que tiene la característica de ser universalizables) y con los que se interpretan los significados morales de las acciones.

La integridad profesional y su contraparte la corrupción profesional nos dicen mucho del modo de ser moral de un profesional. Nos señalan el modo en que la profesionalidad califica un modo de ser completo de un trabajador. Por ejemplo, si alguien conoce los principios y actúa de acuerdo con ellos simplemente para que no se le pille en falta, pensamos que carece de auténtica profesionalidad. No creemos que la profesionalidad sea un mero seguir las reglas, sino que estriba en creer en ellas y en sentirlas como propias. Aunque aquí uno tiende a expresarse en un lenguaje psicológico, lo que este «sentir como propio» abarca es una reflexión moral sobre lo que uno cree y la razones por las que cree que está bien creer en ello. Uno solo es un profesional si ha hecho tal reflexión y el resultado de la misma anima la totalidad de su conducta. En caso contrario, la falta de integridad profesional se muestra en la superficialidad con la que uno cree en los principios profesionales. Si, por ejemplo, a un profesional se le preguntase qué clase de acontecimientos le harían dimitir de un puesto de trabajo y no supiera qué contestar, habría que interpretar que le falta una idea clara de la importancia moral de los compromisos de su profesión $\mathrm{y}$, por tanto, también de su propia identidad como profesional.

El hecho de que la integridad de la que estamos hablando sea la propia de un miembro de una institución profesional nos muestra otro aspecto de la moral profesional. Mientras que la integridad personal tiene los rasgos típicos de una virtud individual, la integridad profesional tiene además los rasgos propios de una virtud social. En la medida en que en su núcleo está la creencia en valores y principios proclamados por la profesión, en los que también creen el resto de los miembros de la profesión y que la sociedad en general acepta, la integridad profesional no es solo una norma de excelencia profesional, sino además un valor relacional que vincula a cada profesional con los demás. Ese vínculo implica la necesidad de sentirse parte de una comunidad que viene definida por la creencia compartida en ciertos principios y también en ciertos modos de proceder en la discusión, el acuerdo y la validación de los mismos. La participación en esta clase de interacciones profesionales, en los procesos en que se concretan y en la evolución propia de las nociones que conllevan, implica una interpenetración entre lo personal y lo profesional que no se da en otras áreas de la vida. Por lo mismo, la falta de integridad y la corrupción profesional pueden comenzar por una falta de vinculación con los miembros de la propia profesión, un «aislamiento» de los demás y una falta de claridad a la hora de concebir la faceta institucional de la propia actividad.

El sentido de la integridad profesional se expresa en ese peculiar conjunto normativo que compone los códigos de deontología profesional. No obstante, como se sigue de lo anterior, se necesita de algo más que el seguir las reglas para actuar de un modo íntegro. Los códigos profesionales no lo garantizan y se requiere que la propia comunidad profesional genere una cultura de la integridad como parte del orgullo de ser profesional. Todas las instituciones necesitan que sus miembros mantengan un alto sen- 
tido de la dignidad profesional como un bastión contra las tentaciones corruptoras. Parece una ley empírica que quienes están identificados con los valores instituciones desarrollan hábitos altruistas y que no suelan considerar que algo menos que lo honesto sea beneficioso. Por el contrario, la falta de identificación profesional puede ser el primer paso de un camino hacia una práctica acomodaticia y, finalmente, corrupta, sobre todo cuando ésta tiene que realizarse en organizaciones ya degradadas por el ejercicio de una autoridad anacrónica y la falta de recursos.

Hay, no obstante, que observar que esta concepción de la moral profesional que subraya el compromiso personal con el cumplimiento de los deberes profesionales como núcleo de la identidad profesional contiene una fuente potencial de conflictos. No me refiero a los conflictos y dilemas éticos que los profesionales tienen que resolver en el ejercicio habitual de la profesión, producto de las exigencias contrapuestas de las muchas expectativas que recaen sobre sus posiciones y funciones en las organizaciones que los emplean. Me refiero a algo de diferente naturaleza. A menos que una persona no tenga más convicciones morales que las convicciones profesionales, se puede ver en situaciones en las que el cumplimiento de los deberes profesionales sea contrario a algunas creencias morales fundamentales. Normalmente se ha tratado este tipo de conflicto como el choque entre los valores personales y los deberes profesionales. El conflicto ha parecido trágico, porque en esos casos o bien uno ha de incumplir las obligaciones que fija la profesionalidad, o bien ha de transgredir principios firmemente sostenidos. Se produce, entonces, una quiebra en el propio sentido de la identidad, dividiéndose en una parte personal y otra profesional. Si trata de salvar su identidad personal, faltará a la confianza de sus clientes y a la de la comunidad profesional. Si trata de ser fiel a su compromiso profesional, faltará a su propio sentido de lo decente. Mientras que todos aspiramos a vivir como nuestro sentido de la identidad requiere y nos concebimos como personas para las que ese modo de vivir es importante, podemos encontrarnos con situaciones en las que tal cosa no sea posible. Entonces, podemos perder el sentido moral de la orientación y, después de la acción, darnos cuenta que hemos sido como nunca habíamos pensado que seríamos.
Naturalmente, las instituciones profesionales sostienen que esta clase de dilemas han de resolverse dando prioridad a los deberes profesionales y excluyendo como privadas las creencias personales. Hay buenas razones para ello. La fundamental se encuentra en la propia protección de los clientes de la arbitrariedad del poder profesional. No obstante, no son menos importantes, desde el punto de vista profesional, el fortalecimiento de la unidad profesional y de la autoridad para definir la identidad profesional. Sin embargo, el precio de excluir como privadas algunas creencias, valores o convicciones que son fundamentales para la persona, representa a veces un coste demasiado alto para que alguien lo pueda asumir sin más. Por eso no es raro que los profesionales decidan que, en tales casos, la integridad personal está por encima de las exigencias profesionales y, por tanto, que lo mejor que pueden hacer es rechazar la prestación del servicio a favor de otro profesional que no tenga los mismos conflictos morales.

Este tipo de elección a favor de la integridad personal, en principio representa una opción admirable, puesto que no implica la infracción de ninguna regla moral fundamental y además cuenta con el valor añadido de la manifestación de un cierto coraje personal para enfrentarse a la situación y hacer una elección difícil. Sin embargo, desde el punto de vista profesional, estas elecciones no están bien vistas, puesto que no es difícil que se interpreten como un desafío a la autoridad moral de la institución profesional, la cual espera que el conjunto de razones que justifiquen cualquier elección profesional sea estrictamente profesional.

De los profesionales se espera que construyan su identidad profesional en torno a la adhesión íntima a los valores profesionales. Ahora bien, un profesional que ha vivido dramáticamente un dilema entre sus convicciones personales y sus convicciones profesionales, es alguien que se ha roto por dentro. El obligarle a considerar lo personal como impropio, puede que no sea la mejor manera de ayudarle a reconstruir una personalidad integrada. Conforme vamos siendo más conscientes de las dimensiones que tiene esta clase de dilemas, más claro se ve que necesitamos de un nuevo sentido de la profesionalidad, el cual sea capaz de admitir como apropiadas las creencias personales. Desde este punto de vista, el buen profesional no sería 
esa especie de autómata que no expresa nada personal en sus relaciones profesionales. Por el contrario, el buen profesional sería aquél capaz de guiarse por un conjunto de valores (ya fueran personales, ya fueran profesionales, la distinción ya no tendría importancia), los cuales estarían justificados por los criterios habituales de equidad y racionalidad que exigimos a los valores públicos. La identidad profesional reconstruida de esta manera no sufriría entonces, cuando se enfrentara a dilemas morales difíciles, y para el profesional tendría menos coste decidirse por una opción. Asimismo, la propia institución profesional podría salir reforzada al aceptar la existencia de tales dilemas y la variedad posible de sus soluciones a la vista de la aplicación no ya de un código de profesionalidad último e indiscutible, sino de aquellos principios que son los que todos utilizamos en la resolución de los problemas morales. La garantía de que seguimos estando en un terreno distintivamente moral sigue estando en la capacidad de nuestro razonamiento de ser universalizable y así compartible por todos aquéllos que, dispuestos a examinar las cosas con la misma voluntad, forman la comunidad profesional.

Para terminar me gustaría traer esta reflexión, quizás demasiado abstracta, a un terreno en el que todo se pueda entender mejor, el de la necesidad de hacer prácticos principios profesionales que no siendo discutibles, su modo de entenderlos por parte del profesional puede ser contrario a un sentido profundo de lo que es la actividad profesional. Me ayudo para ello de un texto ya antiguo, pero que creo conserva toda su fuerza de sugestión.

Ciertamente, existen algunos ensayos que, de vez en cuando, vale la pena releer, porque contienen alguna verdad que es necesario mantener viva a lo largo del tiempo. Éste es el caso del artículo de David Soyer, The Right to Fail, de 1963. Se trata de un artículo que puede parecer anticuado. Escrito en el apogeo del trabajo social de casos, sus referencias teóricas son las obras de Gordon Hamilton y de Helen Harris Perlman. De modo que el mundo profesional en el que se inscribía hace mucho que desapareció. $\mathrm{Y}$, sin embargo, la experiencia que nos transmite sigue siendo memorable:

Con frecuencia, los trabajadores sociales desalentamos en vez de animar; $y$, a veces, defrauda- mos la fe que tenemos en el valor y en la capacidad de los seres humanos, al destruir las ambiciones de nuestros clientes (p. 53).

En su experiencia profesional, Soyer se había encontrado con muchas personas discapacitadas y con problemas de personalidad. Era consciente (porque la ortodoxia del trabajo social de casos así lo repetía una y otra vez) de que el profesional debe representar para su cliente un modo de enjuiciar bien fundado y realista. Él, sin embargo, se había dado cuenta de que en la práctica esa conducta profesional lo que implicaba era que, ante cualquier propuesta inverosímil del cliente, el trabajador social levantara un muro de objeciones para desanimarlo.

Creo que todos conocemos el modo en que se va generando tal conducta. Se reconoce, ciertamente, que es un principio esencial de la práctica de la profesión el respeto al derecho de autodeterminación del cliente. Si bien, también se sabe que no hay que respetarlo cuando, de su ejercicio, se derive un peligro grave para otras personas o para el propio cliente. Luego, quizás, se pase a creer que tampoco habría que respetarlo cuando las decisiones del cliente sean irracionales o erróneas. Por último, se termina adoptando, como una conducta rutinaria, la protección del cliente de cualquier elección que pueda realizar y que, a juicio del trabajador social, pueda implicar una experiencia penosa. El resultado es conocido. Por todas las instituciones sociales se encuentran personas que sufren de esta «sobreprotección» y que, en un sentido, están «institucionalizadas»: «En efecto, una de sus carencias es que nunca han corrido la aventura de someter a la prueba de la realidad sus "sueños de gloria" de adolescentes sin haberlos antes sopesado sensatamente con un trabajador social» (57). Lo que estas personas han perdido es la libertad: la libertad de indagar por sí mismas a dónde llevan los caminos y la libertad de fracasar y de aprender del fracaso.

Soyer recuerda que hay dos muy buenas razones para alentar las ambiciones de los clientes. La primera es muy sencilla: es posible que el cliente acierte y que el profesional se equivoque. La segunda consiste en que,

Sólo a través de la misma vida el cliente puede realmente intentar, comprobar y templar sus posibilidades, sus fantasías y sus metas. Ni toda la charla del mundo acerca de «cabezas» y «pa- 
redes» o acerca de la comparación de la dureza relativa de cabezas y paredes tendrá el mismo efecto en el cliente que el que vaya y se golpee la cabeza con una pared de verdad. Éste es el modo en que las personas crecen y logran adquirir una idea sensata de sí mismas y del mundo. Tienen éxitos y tienen fracasos; y, a través de los éxitos y los fracasos, aprenden (p. 61).

Soyer quiso dejarnos un mensaje claro: el derecho de autodeterminación comprende también el derecho de fracasar. Los profesionales quieren evitar a sus clientes riesgos y situaciones penosas. Con todo, tendrían que entender que el valor de una vida no reside únicamente en el bienestar que se tiene; que su valor depende (y, a veces, de una manera más fundamental) en que la sintamos como fruto de nuestras propias elecciones, las que tienen éxito y también las que son un fracaso. Ese valor es el que protege el derecho de autodeterminación. Y el reconocimiento de ese valor, como señala Soyer, es el que ha dado al trabajo social una fe incomparable en el ser humano.

\section{Nota bibliográfica}

Que la universalizabilidad sea la característica básica del fenómeno moral es un punto de acuerdo de la teoría ética contemporánea, aunque luego las distintas corrientes filosóficas la interpreten de modos diferentes. Puede verse una introducción sencilla al tratamiento de este criterio en J. Rachels (2003) y una discusión más compleja en T. Jollimore (2005). Las opiniones que aquí expreso deben mucho a los trabajos clásicos de R. M. Hare (1952, 1981, 1997) y J. C. Harsanyi (1982).

La diferencia que existe entre los juicios de ética profesional y los juicios de responsabilidad profesional, cuya naturaleza es jurídica, he tratado de explicarla en D. Salcedo (2012). En este mismo trabajo aparece una concepción novedosa del fenómeno moral de la corrupción profesional. Sobre la naturaleza institucional de la ética profesional puede verse D. Salcedo (2010).

Los problemas filosóficos que presenta la integridad moral son difíciles y variados. He tratado de extender algunas de las ideas que se encuentran en la ya amplia literatura sobre este tópico al ámbito de la integridad profesional. La deuda mayor la tengo con los trabajos de L. McFall (1987) y Ch. Calhoun (1995). Puede verse una discusión actualizada de las distintas concepciones sobre el asunto en D. Cox, et al. (2011).

Las discusiones sobre el papel de los valores personales en las actividades profesionales son antiguas. En el ámbito del trabajo social, quizás el trabajo más clásico sea el de C. S. Levy (1976). La posición ortodoxa sobre el modo de resolver estos conflictos se puede ver en $\mathrm{N}$. Leighton (1985), N. Linzer (1999) y F. G. Reamer (1995). En D. Salcedo (2013) he analizado esta clase de dilemas en el marco de las concepciones sobre la identidad profesional. Para ello me he aprovechado de la discusión que tuvo lugar en las páginas de la revista electrónica Journal of Social Work Values and Ethics, entre los años 2007 y 2010, entre Richard Spano y Terry Koenig (2007, 2008, 2009 y 2010), por un lado, y Paul Adams (2008, 2009a y 2009b), por otro. Aunque moldeados en un ámbito jurídico, mis opiniones deben mucho a los análisis de A. I. Applbaum (1993).

La referencia completa al trabajo de D. Soyer (1963) aparece en la bibliografía. Sobre las dificultades que plantean los conceptos de autonomía y autodeterminación en la práctica profesional me permito remitir a mis trabajos D. Salcedo (2000, 2001).

\section{Referencias bibliográficas}

Adams, P. (2008). The Code of Ethics and the Clash of Ortodoxies: a Response to Spano and Koenig. Journal of Social Work Values and Ethics, 5(2).

Adams, P. (2009a). Code of Duties of Ideological Club? Journal of Social Work Values and Ethics, 6(1).

Adams, P. (2009b). Letter to the Editor. Limits of a Code of Ethics. Journal of Social Work Values and Ethics, 6(3).

Adams, P. (2009c). Ethics with Character: Virtues and the Ethical Social Worker. Journal of Sociology and Social Welfare, 36(3), 83-105.

Adams, P. (2011). Coercing Conscience: Professional Duty or Moral Integrity». Journal of Social Work Values and Ethics, 8(1). 
Applbaum, A. I. ([1993], 1999). The Remains of the Role. En A. I. Applbaum, Ethics for Adversaries. The Morality of Roles in Public and Professional Life. Princeton, N. J.: Princeton U. P.

Calhoun, Ch. (1995). Standing for Something. Journal of Philosophy, XCII, 235-60.

Cox, D., et al., (2011). Integrity. En E. N. Zatta (ed.), Stanford Encyclopedia of Philosophy. Recuperado de: http://plato.stanford.edu/entries/integrity/.

Hare, R. M. ([1952], 1975). El lenguaje de la moral, México: UNAM.

Hare, R. M. (1981). Moral Thinking. Its Levels, Method and Point. Oxford: Clarendon P.

Hare, R. M. ([1997], 1999). Ordenando la ética. Una clasificación de las teorías éticas. Barcelona: Ariel.

Harsanyi, J. C. ([1982], 2004). La moral y la teoría de la conducta racional. En F. Lara y P. Francés (eds.). Etica sin dogmas. Racionalidad, consecuencias y bienestar en el utilitarismo contemporáneo. Madrid: Biblioteca Nueva.

Jollimore, T. (2005). Impartiality. En E. N. Zatta (ed.), Stanford Encyclopedia of Philosophy. Recuperado de: http://plato.stanford.edu/entries/impartiality/.

Leighton, N. (1985). Personal and Professional Values - Marriage or Divorce? En D. Watson (ed.), A Code of Ethics for Social Work. The Second Step. Londres: Routlege \& K. Paul.

Levy, C. S. (1976). Personal versus Professional Values: The Practitioner's Dilemma. Clinical Social Work Journal, 4(2), 110-120.

Linzer, N. (1999). Resolving Ethical Dilemmas in Social Work Practice, Boston: Allyn and Bacon.

McFall, L. (1987). Integrity. Ethics, 98, 5-20.

Rachels, J. ([2003], 2007). Introducción a la filosofía moral. México: F.C.E.

Reamer, F. G. (1995). Social Work Values and Ethics. Nueva York: Columbia U. P.

Salcedo Megales, D. (2000). El respeto de la autonomía personal y la definición del paternalismo profesional. En Ma José Martínez (comp.). Para el trabajo social. Aportaciones teóricas y prácticas. Granada: Maristán.

Salcedo Megales, D. (2001). Autonomía y bienestar. La ética del Trabajo Social. Granada: Comares.

Salcedo Megales, D. (2010). Los fundamentos normativos de las profesiones y los deberes de los trabajadores sociales. Trabajo Social Global, 1(1), 10-38.

Salcedo Megales, D. (2012). Mala praxis, corrupción y juicios de ética profesional. Las Torres de Lucca. Revista Internacional de Filosofia Política, 1, 91-122.

Salcedo Megales, D. (2013). La moral personal, la profesional y la política en el ámbito del trabajo social. En L. M. Rondón y M. L. Taboada (comps.). Voces para la ética del trabajo social. Madrid: Paraninfo.

Soyer, D. ([1963], 1975). The Right to Fail. En F. E. McDermott (ed.). Self-Determination in Social Work, Londres -Boston: Routledge \& K. Paul.

Spano, R. y Koenig, T. (2007). What Is Sacred When Personal and Professional Values Collide? Journal of Social Work Values and Ethics, 4(3).

Spano, R. y Koenig, T. (2008). A Response to Paul Adams. The Code of Ethics and the Clash of Ortodoxies. Journal of Social Work Values and Ethics, 5(2).

Spano, R. y Koenig, T. (2009). Free Speech and Special Responsibilities of the Profession. Journal of Social Work Values and Ethics, 6(1).

Spano, R. y Koenig, T. (2010). A Reply to Adams: the Delicate Balance Between Private Viewpoints and Professional Duties. Journal of Social Work Values and Ethics, 7(1). 